\title{
COME CATTURARE NEL TEMPO GLI EVENTI PRIMARI DI PROCESSI NATURALI: I LASER AD IMPULSI ULTRABREVI
}

\author{
Nota del s.c. SANDRO DE SILVESTRI e del m.e. ORAZIO SVELTO (*)
}

(Adunanza del 15 novembre 2012)

\begin{abstract}
SUNTO. - Il presente lavoro presenta una breve rassegna delle moderne tecnologie laser che consentono di generare impulsi di luce della durata fino a "pochi cicli ottici" su ampi intervalli spettrali, che permettono di filmare l'evoluzione di processi dinamici straordinariamente brevi. In particolare verranno illustrati i risultati ottenuti dallo studio degli eventi primari di due processi importanti in natura che coinvolgono l'assorbimento della luce, quali la visione e la fotosintesi. Questi eventi avvengono in tempi molto rapidi e innescano, nei due casi, l'intero processo. Lo studio di questi eventi è importante sia per ragioni di carattere fondamentale sia al fine di progettare sistemi molecolari artificiali, di elevata efficienza, da impiegare per nuovi dispositivi nel campo della visione artificiale e del fotovoltaico.
\end{abstract}

ABSTRACT. - This paper presents a brief review of the modern laser technologies that enable to generate light pulses lasting "a few optical cycles" and tunable over large spectral ranges, thus allowing to follow the evolution of extraordinarily fast dynamical processes. In particular, we will describe the results obtained from the studies of the primary events of two important processes in nature involving the absorption of light, namely vision and photosynthesis. These events last for extremely short times and trigger the corresponding overall processes. The study of these events is important both for fundamental reasons and for the design of artificial molecular systems

Milano, Italy.

Dipartimento di Fisica, Politecnico di Milano, Piazza L. da Vinci 32, 20133

E-mail: orazio.svelto@polimi.it ; sandro.desilvestri@polimi.it 
of high efficiency to be used for new devices in the field of artificial vision and of photovoltaics.

\section{INTRODUZIONE}

L'osservazione e lo studio dell'evoluzione temporale di un processo naturale ha sempre presentato aspetti di grande interesse scientifico ed applicativo: infatti, si ha così la possibilità non solo di far luce sui relativi meccanismi elementari ma anche di intervenire e guidare l'evoluzione stessa. Tuttavia, dall'invenzione dei primi orologi meccanici, avvenuta nel XVI secolo, fino al XIX secolo, la nostra capacità investigativa era limitata a quei processi che avvenivano su scale temporali compatibili con la nostra percezione sensoriale diretta. Nel caso dell'occhio, la persistenza delle immagini sulla retina $(\approx 0.1 \mathrm{~s})$ impedisce, infatti, di studiare fenomeni più rapidi. A partire dal XIX secolo, diverse evoluzioni tecnologiche hanno permesso di ridurre drasticamente gli intervalli temporali misurabili.

Utilizzando diverse macchine fotografiche lungo il percorso di un cavallo al galoppo, dotate di otturatori meccanici molto rapidi (circa 1 millisecondo) e con un ingegnoso sistema di sincronizzazione, E. Muybridge riuscì nel 1878 ad analizzare le varie fasi del galoppo. Dimostrò, in questo modo, che, per un breve istante invisibile all'occhio umano, l'animale teneva tutti e quattro gli zoccoli sollevati dal suolo. Il quesito se ci fosse un determinato instante in cui un cavallo, durante il suo galoppo, tenesse le sue quattro zampe staccate dal suolo appassionava infatti parecchi esperti del settore di quel tempo. L'interesse era tale che L. Stanford, il fondatore negli anni successivi dell'Università di Stanford, istituì un grosso premio per chi fosse riuscito a risolvere questo quesito. Muybridge, con le sue ingegnose misure, riuscì ad aggiudicarselo.

La "fotografia a flash" utilizza lampi di luce molto brevi per illuminare un oggetto, consentendo di congelarne una fase del movimento: usando una serie di flash nel tempo che illuminano sequenzialmente lo stesso oggetto e combinando i singoli fotogrammi in una sorta di "moviola", è possibile ottenere una riproduzione completa del moto. Il corrispondente strumento, detto stroboscopio e inventato nel 1930, portò la risoluzione temporale ad alcuni microsecondi: risalgono a questo periodo 
le famose fotografie del proiettile che attraversa una mela ad opera di $\mathrm{H}$. Edgerton. Un tempo di esposizione dell'ordine del microsecondo rappresenta il limite ottenibile con le sorgenti di luce ordinarie.

Un radicale miglioramento della risoluzione temporale è stato reso possibile dall'invenzione del laser, nel 1960. Qualche anno dopo questa invenzione e con una tecnica denominata mode-locking, fu infatti possibile generare, da un laser, impulsi di luce di alcuni picosecondi $\left(1 \mathrm{ps}=10^{-12} \mathrm{~s}\right.$, il prefisso pico deriva dalla parola italiana piccolo). Attualmente, le sorgenti laser sono in grado di generare direttamente impulsi luminosi con durate sino ad alcune decine di femtosecondi $\left(1 \mathrm{fs}=10^{-3} \mathrm{ps}=10^{-15} \mathrm{~s}\right.$, il prefisso femto deriva dal danese o norvegese femten che significa quindici).[1] Inoltre, il loro utilizzo, combinato con tecniche basate su processi ottici non lineari, ha consentito di produrre impulsi ancora più brevi, da pochi femtosecondi,[2] sino ad arrivare attualmente ad impulsi della durata di un centinaio di attosecondi $\left(1\right.$ as $=10^{-3} \mathrm{fs}=10^{-18} \mathrm{~s}$, il prefisso atto deriva dal danese atten che significa diciotto).[3] Tali impulsi di luce consentono di estendere oltremisura la nostra capacità di osservare in tempo reale la dinamica di processi naturali.

Esistono in natura eventi tanto rapidi da richiedere un'elevata risoluzione temporale? La risposta è affermativa per numerosi fenomeni a livello atomico e molecolare. Quando una corrente elettrica fluisce in un filo di rame, gli elettroni urtano contro gli atomi del reticolo mediamente ogni 10 fs. Per leggere questo articolo, i recettori della retina (molecole di rodopsina) ripetono per circa 10 miliardi di volte al secondo una foto-reazione il cui evento primario è concluso in soli 200 fs. Numerosi processi di rilassamento dell'energia assorbita da parte delle molecole avvengono su scale dalle decine alle centinaia di femtosecondi. I moti dei nuclei e degli elettroni all'interno di una molecola avvengono su una scale dei tempi comprese rispettivamente tra un centinaio di femtosecondi e pochi attosecondi. In termini di meccanica quantistica, si può stabilire un'analogia fra l'evoluzione temporale di una opportuna sovrapposizione coerente di stati stazionari di una data particella (es. un nucleo o un elettrone) e il moto classico di quella particella. Supponiamo ad esempio che una particella si trovi in uno stato non stazionario dato dalla sovrapposizione coerente di uno primo stato di energia $E_{1}$ e di un secondo stato di energia $E_{2}\left(E_{2}>E_{1}\right)$. Tale sovrapposizione costituisce un pacchetto d'onda. Risolvendo l'equazione di Schrödinger per la funzione d'onda della particella, si ricava che la corrispondente distribuzione di probabilità nello spazio oscilla con un periodo $T=h /\left(E_{2}-E_{1}\right)$, dove $h$ è la costante 
di Planck ( $h=6.63 \times 10^{-34} \mathrm{~J} \mathrm{~s}$ ). La variazione della posizione del centro di massa del pacchetto d'onda costituisce l'analogo quantistico del moto classico della particella in considerazione. Pertanto, quanto maggiore è la differenza in energia, $\Delta E=E_{2}-E_{1}$, fra i due stati coinvolti, tanto più veloce è il moto della particella. Ad esempio, nel caso dei moti vibrazionali degli atomi all'interno di una molecola, la differenza di energia fra i livelli vibrazionali è dell'ordine del millielettronvolt $\left(1 \mathrm{eV}=1.6 \times 10^{-19} \mathrm{~J}\right)$ e pertanto il corrispondente moto avviene su scale temporali che vanno dalle decine alle centinaia di femtosecondi. Il moto degli elettroni in nanostrutture o all'interno di una molecola avviene invece su scale temporali ancora più brevi, dalle decine di femtosecondi a pochi attosecondi. Infine, dinamiche ancora più veloci avvengono all'interno del nucleo, dove le differenze tra i livelli energetici sono dell'ordine di $10^{6} \mathrm{eV}$ : in questo caso il tempo caratteristico per il movimento dei nucleoni all'interno del nucleo risulta dell'ordine di alcuni zeptosecondi $\left(1 \mathrm{zs}=10^{-3}\right.$ as $=10^{-21} \mathrm{~s}$; il prefisso zepto deriva dal francese sept, sette, che richiama il fatto che 10${ }^{21}$ può essere scritto come la settima potenza di un millesimo, $1 / 1000^{7}$ ). Lo studio dei fenomeni ultrabrevi è dunque un argomento interdisciplinare, che riguarda sia le scienze di base (fisica, chimica, biologia) sia le discipline applicate (elettronica, fotonica).

Nel seguito verranno illustrati i risultati ottenuti dallo studio di due processi importanti in natura, che coinvolgono l'assorbimento della luce, come la visione e la fotosintesi, determinanti per l'esistenza del mondo animale e vegetale, rispettivamente. Tali processi sono innescati da eventi primari molto rapidi nel tempo. Questo è un modo per la natura di garantirsi la massima efficienza nello sviluppo del processo stesso in quanto la brevità dell'evento primario riduce drasticamente la competizione con altri processi dissipativi. Per poter catturare nel tempo questi eventi è necessario disporre di impulsi estremamente brevi, cioè caratterizzati da una durata di alcuni femtosecondi, e accordabili, cioè in grado di ricoprire un ampio intervallo spettrale nel visibile-ultravioletto in modo da poter entrare in risonanza con gli stati elettronici delle molecole coinvolte in questo processo. La tecnica ottica generalmente usata per studiare questi processi dinamici estremamente veloci è detta di "pompa e sonda". Un primo impulso, detto di "pompa", con il suo spettro posizionato nella regione di assorbimento del sistema coinvolto eccita il sistema stesso e dà inizio al fenomeno che si vuole studiare (reazione chimica, rilassamento energetico, cambiamento strutturale). Un secondo impulso, detto di "sonda", sincronizza- 
to con il primo e opportunamente ritardato, rivela l'evoluzione del sistema. La dinamica del sistema viene misurata tramite la variazione della trasmissione dell'impulso di sonda. A ciascun ritardo tra pompa e sonda corrisponde un "fotogramma"; anche qui, combinando i singoli fotogrammi si può ottenere una sorta di film dell'evoluzione temporale del sistema. Si noti che lo studio dei processi di visione e fotosintesi è importante non solo per ragioni di carattere fondamentale ma anche per il fatto che tali studi potranno servire a progettare sistemi molecolari artificiali, di elevata efficienza, da impiegare per nuovi dispositivi nel campo della visione artificiale e del fotovoltaico.

\section{GENERAZIONE DI IMPULSI DI LUCE ULTRABREVI “ACCORDABILI”}

Impulsi ultrabrevi di pochi cicli ottici nel visibile (corrispondenti ad una durata inferiore alla decina di femtosecondi) vengono prodotti e amplificati partendo da una sorgente laser dotata di impulsi più lunghi pari a un centinaio di femtosecondi (generalmente una laser a Titanio in zaffiro che opera alla lunghezza d'onda di $800 \mathrm{~nm}$ ). Lo schema di principio dell'intero sistema, basato sulla combinazione dei processi di "amplificazione parametrica" e "compressione ottica",[4] è mostrato in Fig. 1.

Dapprima, l'impulso proveniente dalla sorgente laser viene inviato in un opportuno sistema che produce, mediante processi nonlineari, una modulazione della fase dell'impulso stesso (automodulazione di fase, Fig. 1). All'uscita di questo modulatore, l'impulso di luce è quindi caratterizzato da uno spettro più largo rispetto a quello dell'impulso in ingresso. Se l'allargamento è sufficientemente elevato da ricoprire l'intero spettro del visibile, questi impulsi sono detti a luce bianca poiché di questo colore appare il corrispondente fascio all'occhio umano. Tali impulsi tuttavia sono generalmente di bassa energia (decine di nanojoule) rendendo così necessaria una loro amplificazione ai fini dell'utilizzo in un successivo apparato di misura. L'impulso viene pertanto inviato ad un successivo stadio di amplificazione (amplificatore parametrico, Fig. 1), dove guadagna energia a spese di un intenso impulso di luce laser violetta, ottenuto dalla sorgente di partenza mediante generazione di seconda armonica. Il processo di amplificazione avviene facendo interagire i due impulsi all'interno di un opportuno cristallo, dove l'impulso violetto trasferisce energia all'impulso di luce bianca mediante un processo ottico non-lineare noto con il nome di "amplificazione parametrica": l'energia 
dell'impulso di luce bianca può così aumentare in maniera notevole (anche di un fattore 104). Da notare inoltre che, se solo una parte dello spettro dell'impulso di luce bianca proveniente dall'automodulatore viene selezionata per il suo invio all'amplificatore, il processo di amplificazione genererà impulsi energetici solo in quella regione spettrale. Cambiando il centro dello spettro selezionato è possibile così ottenere impulsi di luce sufficientemente energetici e accordabili in frequenza.

L'impulso amplificato viene quindi inviato ad un opportuno compressore (Fig. 1) il quale non fa altro che convertire la modulazione di fase dell'impulso in modulazione di ampiezza. In uscita dal compressore è allora possibile ottenere impulsi di luce che, sulla base del noto teorema di Fourier, siano pari all'inverso della banda dell'impulso. Poiché questa banda era stata notevolmente allargata mediante l'automodulazione di fase, in uscita da questo sistema si ottengono impulsi notevolmente più brevi di quelli emessi dalla sorgente laser originaria e sufficientemente energetici per le applicazioni. Nel caso di compressione ottimale, si possono così ottenere impulsi con durata pari a pochi femtosecondi (corrispondenti nello spettro visibile a pochi cicli ottici dell'onda portante). Inoltre, come spiegato in precedenza, questi impulsi possono essere accordati, col loro centro-banda, in tutta la regione del visibile o anche nell'infrarosso.

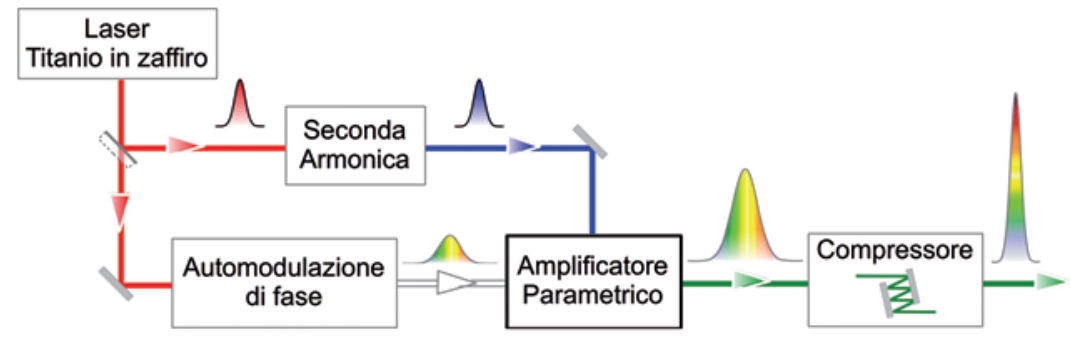

Fig. 1 - Schema di principio per la generazione di impulsi ultrabrevi e accordabili.

\section{IL PROCESSO PRIMARIO NELLA VISIONE}

La superficie sensibile dell'occhio, la retina, è costituita da fotorecettori (bastoncelli e coni) il cui compito è quello di trasformare in impulsi elettrici le informazioni ricevute dalle reazioni fotochimiche che vengono attivate dalla radiazione luminosa. Questi impulsi vengono 
poi inviati ai neuroni retinici per una prima elaborazione del segnale visivo. Coni e bastoncelli hanno una diversa sensibilità spettrale e diverso adattamento al buio. I bastoncelli sono circa 1000 volte più sensibili alla luce dei coni e molto più numerosi (rispettivamente 120 milioni contro 6 milioni). In maniera approssimativa, si può dire che di notte vediamo con i bastoncelli e di giorno con i coni. Coni e bastoncelli contengono rispettivamente la iodopsina e la rodopsina, che sono tasche proteiche, costituite da diverse forme dell'opsina (una proteina) al cui interno è presente un cromoforo detto retinale, la cui struttura chimica è quella di un'aldeide della vitamina $A$, responsabile dell'assorbimento della luce. L'evento primario della visione consiste in una reazione di foto-isomerizzazione del retinale. Al buio il retinale è in conformazione 11-cis, in cui cioè un doppio legame in posizione $n=11$ della catena $[(\mathrm{C}-\mathrm{H})=]_{n}$ che costituisce la molecola di retinale è in posizione ripiegata (Fig. 2b). Se ora il retinale assorbe un fotone esso può diventare retinale tutto-trans, in cui cioè lo stesso legame passa a una forma distesa (trans) (Fig. 2d). Questa piccola modifica strutturale del retinale comporta la scissione del complesso retinale-opsina ed è in grado di cambiare la conformazione sterica della tasca proteica della opsina. La tasca proteica così attivata dà luogo ad una serie di ulteriori reazioni che avviano la formazione e la trasmissione di un segnale elettrico. Poiché la molecola di retinale, come detto, è presente sia nei coni che nei bastoncelli, il meccanismo di attivazione è del tutto simile per entrambi. La diversa sensibilità e la diversa regione spettrale di funzionamento sono essenzialmente determinate, nei due casi, da due diverse conformazioni del legame opsina-retinale che determinano la regione spettrale di assorbimento del retinale stesso. In ambedue i casi, un enzima specifico produce poi la riconversione del retinale nell'isomero 11-cis e con questo la sua ricombinazione con l'opsina; il pigmento visivo viene così continuamente rigenerato.

Lo studio della dinamica della conversione del cromoforo retinale 11-cis alla sua forma tutto-trans è stato oggetto di diversi lavori sia teorici che sperimentali, in particolare nella rodopsina. L'elevata resa quantica $(65 \%)$ del processo di trasformazione della rodopsina, che è la ragione dell'elevatissima sensibilità dell'occhio umano, suggeriva infatti la presenza di una fotoreazione insolitamente veloce e unidirezionale: infatti, solo con una reazione molto veloce il processo di fotoisomerizzazione poteva prevalere rispetto agli altri inevitabili processi di dissipazione dell'energia assorbita, disponibili per la stessa molecola. La presenza di una 
reazione molto veloce nella variazione di conformazione del retinale è stata generalmente attribuita ad una intersezione tra la superficie di energia potenziale dello stato eccitato del retinale in conformazione 11cis con la superficie di energia potenziale dello stato fondamentale della molecola nello stato tutto-trans. L'intersezione viene detta "intersezione conica" poiché, nello spazio tridimensionale delle superfici di potenziale, essa produce un cono. Come i "buchi neri" sono delle singolarità che mettono probabilmente in collegamento due diversi spazi del mondo interstellare, così le intersezioni coniche possono essere viste come "buchi neri della chimica", singolarità che mettono in collegamento due stati elettronici diversi della materia. Inoltre, al pari dei buchi neri interstellari, i buchi neri della chimica erano previsti solo teoricamente e risultavano di difficile osservazione sperimentale. Ottenere prove sperimentali dirette del coinvolgimento di una intersezione conica era infatti impegnativo: l'intervallo di energia tra gli stati elettronici della molecola che reagisce cambia in modo notevole su una scala temporale ultrabreve, il che richiede metodi di osservazione che combinano alta risoluzione temporale e osservazione su un'ampia finestra spettrale. Avendo a disposizione impulsi di luce ultrabrevi e accordabili dal vicino infrarosso al visibile con una risoluzione temporale di circa 10 -fs è stato possibile seguire in modo completo tutta la dinamica temporale di trasformazione del retinale attraverso l'intersezione conica, dimostrando la fondatezza delle previsioni teoriche $\mathrm{e}$, in particolare, una scala dei tempi insolitamente veloce.

La tecnica sperimentale di "pompa e sonda" utilizza un impulso di pompa della durata di 10 fs alla lunghezza d'onda di $500 \mathrm{~nm}$, che viene assorbito dallo stato fondamentale del retinale in configurazione cis. Lo stato dinamico del retinale è poi monitorato mediante impulsi di sonda, opportunamente ritardati rispetto all'impulso di pompa, che coprono un intervallo spettrale $500-750 \mathrm{~nm}$ (7 fs) e 820-1020 nm (13 fs) rispettivamente. In Fig. $2 a$ viene mostrata la reazione di isomerizzazione così come viene direttamente visualizzata dalla misura di "pompa e sonda". A seguito dell'assorbimento dell'impulso di pompa (eccitazione, fase 1), il retinale si porta sulla superficie di energia potenziale corrispondente al primo stato eccitato, dove ha inizio un processo di rilassamento verso il corrispondente minimo di energia potenziale. Tale processo è stato seguito in tempo reale analizzando il retinale eccitato con impulsi di sonda a diversi ritardi in tempo nella regione spettrale 800-1000 nm. Essendo il retinale in stato eccitato questi impulsi di 
sonda provocano un processo di emissione stimolata nel retinale, con conseguente amplificazione dell'impulso di sonda stesso dopo l'attraversamento del retinale. A ritardi crescenti dell'impulso di sonda rispetto a quello di eccitazione, l'amplificazione avviene a lunghezze d'onda sempre più elevate. Questo è indice dell'esistenza di un processo di rilassamento del retinale verso il minimo della curva di energia potenziale dello stato eccitato (Fig. 2a). Utilizzando impulsi di sonda ancora più ritardati nel tempo si è osservato invece un assorbimento del retinale a lunghezze d'onda via via decrescenti nella regione spettrale 800-500 $\mathrm{nm}$. Tale fenomeno è dovuto al fatto che, a causa dell'intersezione conica della curva che descrive lo stato eccitato del retinale 11-cis con quella dello stato fondamentale del retinale all-trans, (Fig. 2a, fase 2), il retinale si è ora portato in quest'ultimo stato. L'assorbimento (assorbimento fotoindotto) a lunghezze d'onda via via decrescenti dell'impulso di sonda deriva dal fatto che il retinale in configurazione all-trans si porta, col tempo, verso il minimo di potenziale del suo stato fondamentale (Fig. 2a, fase 3). L'intero processo di isomerizzazione, dalla fase 1 alla fase 3 si completa in $200 \mathrm{fs}$ come riportato nel lavoro [5] con uno studio di immagini spettrali intervallate di un tempo incredibilmente breve (circa 10 fs) e su un ampio intervallo spettrale (500-1000 nm). Ciò ha consentito di dimostrare sperimentalmente, per la prima volta, l'esistenza di una intersezione conica e di osservare la corrispondente dinamica ultrarapida.
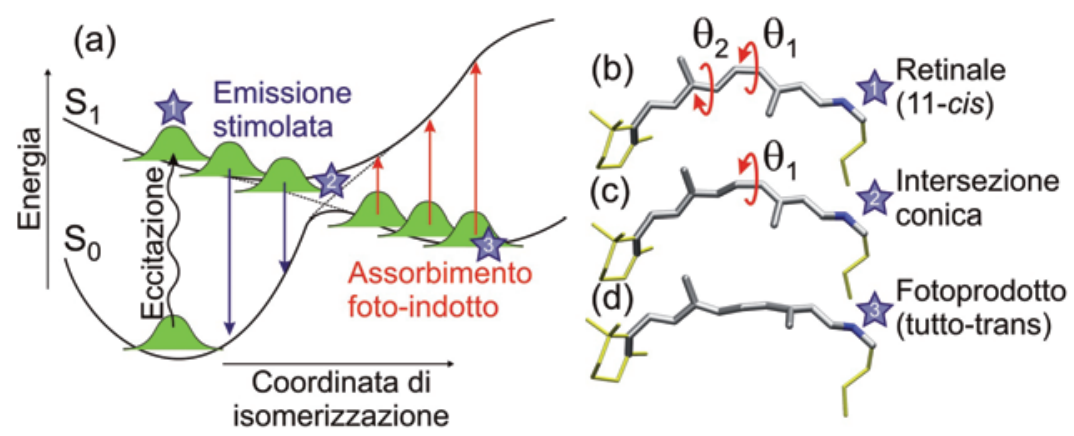

Fig. 2 - (a) Misura della dinamica di isomerizzazione del retinale lungo la coordinata di isomerizzazione: l'eccitazione è indotta dall'impulso di pompa mentre l'emissione stimolata e l'assorbimento sono indotti dagli impulsi di sonda ritardati nel tempo

(fasi 1-3); (b-d) trasformazione della struttura del retinale da cis a tutto-trans durante le tre fasi $\left(\theta_{1}\right.$ e $\theta_{2}$ rappresentano gli angoli di rotazione a cui sono soggetti i legami durante la trasformazione). 


\section{IL PROCESSO PRIMARIO NELLA FOTOSINTESI}

In questo paragrafo viene presentato lo studio di alcuni processi ultrabrevi che coinvolgono una molecola coniugata, il beta-carotene, una catena di atomi di carbonio e idrogeno di eccezionale importanza in fisica, chimica e biologia. In generale le catene coniugate di atomi di carbonio dette carotenoidi sono presenti dovunque nel nostro universo (si trovano anche nello spazio interstellare), danno il colore a molti fiori, frutti o ortaggi comuni, e svolgono funzioni vitali nella fotosintesi delle piante e dei batteri. Nei sistemi fotosintetici la funzione primaria dei carotenoidi è di antenna per la radiazione solare. Queste molecole hanno infatti un forte assorbimento nella regione del visibile (bluverde) e trasferiscono poi l'energia raccolta dal sole ad altre molecole (clorofille) per continuare la fotoreazione. Esse pertanto contribuiscono in maniera decisiva all'efficienza del processo di raccolta di energia solare da parte degli organismi fotosintetici. Il complesso antenna (denominato LH2) è costituita da 9 unità fotosintetiche identiche, ciascuna costituita da un carotenoide e da tre clorofille (BChl, Fig. 3).

Un esempio importante di sistema fotosintetico è rappresentato da alcune alghe, quali i dinoflagellati, che si trovano nelle profondità degli oceani, assorbono una rilevante frazione della luce solare ivi presente e costituiscono un importante anello della catena alimentare; tali alghe utilizzano in prevalenza i loro carotenoidi che, come detto in precedenza, assorbono la componente blu-verde della luce solare che è quella che risulta trasmessa più efficientemente dall'acqua. I complessi antenna di queste alghe sono dei sistemi modello della reazione di fotosintesi e quindi vengono molto studiati. Utilizzando uno di questi complessi antenna e la tecnica di "pompa e sonda", è stato possibile filmare l'evoluzione del trasferimento dell'energia assorbita dalle molecole di beta-carotene verso le clorofille.[6] Un primo impulso di pompa viene assorbito dal beta-carotene e porta la molecola dallo stato fondamentale $\mathrm{S}_{0}$ allo stato eccitato $\mathrm{S}_{2}$ (Fig. 3). Da qui ha inizio una serie di trasformazioni riassunte schematicamente nella citata figura: un rapidissimo passaggio dallo stato eccitato $S_{2}$ allo stato $Q_{x}$ della clorofilla con una costante di tempo di circa $50 \mathrm{fs}$, un rilassamento interno verso lo stato $S_{1}$ in 100 fs seguito da un trasferimento verso lo stato eccitato $Q_{y}$ della clorofilla (con un tempo di trasferimento di 3 ps) oppure, in parallelo, da un rilassamento interno del carotenoide verso il suo stato fondamentale $\mathrm{S}_{0}$ (con un tempo di decadimento di 5 ps). Queste trasformazioni 
vengono visualizzate inviando impulsi di sonda opportunamente ritardati in tempo che vengono assorbiti dagli stati eccitati del beta-carotene. Confrontando misure di "pompa e sonda" del beta-carotene in soluzione e del beta-carotene legato al complesso antenna è possibile determinare gli effetti sui tempi di vita degli stati eccitati dovuti al trasferimento di energia verso le clorofille (e ottenere così la mappa dei tempi riportata in Fig. 3) e valutare l'efficienza di tale trasferimento. In particolare, è stato per la prima volta evidenziato che il trasferimento dallo stato eccitato $\mathrm{S}_{2}$ del carotenoide allo stato eccitato $Q_{x}$ della clorofilla avviene in un tempo veramente molto rapido (50 fs) il che rende conto della elevata efficienza del trasferimento stesso e quindi dell'importanza della presenza del carotenoide nel sistema antenna.
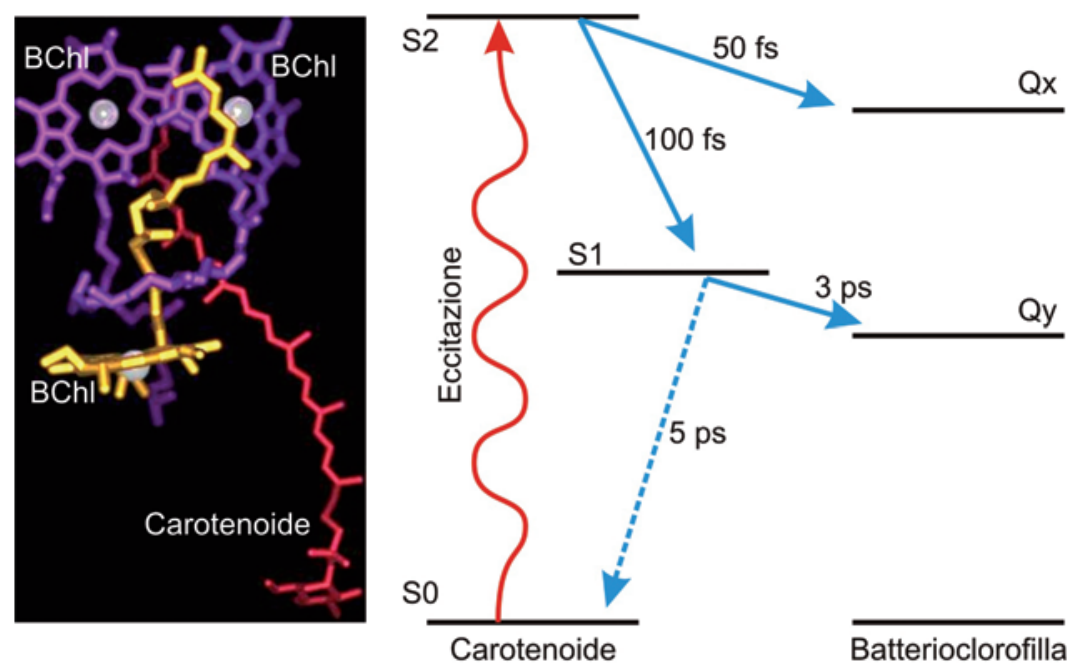

Fig. 3 - A sinistra, uno dei nove elementi del complesso antenna LH2 costituito da un carotenoide e da tre batterioclorofille (BChl); a destra, schema dei livelli elettronici del carotenoide e della batterioclorofilla e relativi tempi di rilassamento e trasferimento di energia.

\section{CONCLUSIONI}

In questa breve rassegna, abbiamo visto come le moderne tecnologie ottiche consentano di generare impulsi di luce della durata fino a "pochi cicli ottici" e che possono ricoprire ampi intervalli spettrali. Questi impulsi, unitamente a particolari tecniche di misura "pompa e 
sonda" consentono di filmare l'evoluzione di processi dinamici straordinariamente brevi. La conoscenza profonda dei meccanismi alla base dei sensori naturali di luce ci aiuterà, in futuro, a progettare congegni artificiali altrettanto efficienti. Le applicazioni sono molteplici, e vanno da nuovi tipi di memorie ottiche, a motori molecolari azionati dalla luce che alimentano nano-dispositivi, fino ad arrivare, in prospettiva, a congegni artificiali fotosensibili che riproducano il comportamento della retina. Ovviamente le possibili applicazioni non si limitano ai soli casi riportati, ma coprono una più vasta gamma di settori dalla fisica della materia, alla chimica e alla biologia. Si tratta del frutto di una evoluzione tecnologica che vede impegnati numerosi gruppi di ricerca in campo internazionale e che pone l'Italia tra i paesi più avanzati in questo settore. In particolare presso il Dipartimento di Fisica del Politecnico di Milano opera una infrastruttura laser, il "Centre for Ultrafast Science and Biomedical Optics” (CUSBO), riconosciuta dall'Unione Europea. Ad essa possono accedere gruppi di ricerca italiani e stranieri che intendono utilizzare la strumentazione avanzata e le tecniche sofisticate di misura, ivi disponibili e in parte descritte nel presente contributo.

\section{BIBLIOGRAFIA}

[1] O. Svelto, "Principles of lasers", $5^{\text {th }}$ ed., Springer (2010).

[2] M. Nisoli, S. De Silvestri, O. Svelto, R. Szipöcs, K. Ferencz, Ch. Spielmann, S. Sartania, F. Krausz, "Compression of high-energy laser pulses below $5 \mathrm{fs}$ ", Opt. Lett. 22 (1997) 522-524.

[3] G. Sansone, E. Benedetti, F. Calegari, C. Vozzi, L. Avaldi, R. Flammini, L. Poletto, P. Villoresi, C. Altucci, R. Velotta, S. Stagira, S. De Silvestri, M. Nisoli, "Isolated single-cycle attosecond pulses", Science 314 (2006) 443-446.

[4] D. Brida, C. Manzoni, G. Cirmi, M. Marangoni, S. Bonora, P. Villoresi, S. De Silvestri and G. Cerullo, "Few-optical-cycle pulses tunable from the visible to the mid-infrared by optical parametric amplifiers", J. Opt. 12 (2010) 013001 (13pp).

[5] D. Polli, P. Altoè, O. Weingart, K.M. Spillame, C. Manzoni, D. Brida, G. Tomasello, G. Orlandi, P. Kukura, R.A. Mathies, M. Garavelli, G. Cerullo, "Conical intersection dynamics of the primary photoisomerization event in vision", Nature 467 (2010) 440-443.

[6] D. Polli, G. Cerullo, G. Lanzani, S. De Silvestri, H. Hashimoto, R.J. Cogdell, "Carotenoid-Bacteriochlorophyll energy transfer in LH2 complexes studied with 10-fs time resolution”, Biophys. J. 90 (2006) 2486-2497. 